\title{
Stochastic economic analysis of dairy cattle artificial insemination reproductive management programs
}

\author{
N. J. Olynk ${ }^{1}$ and C. A. Wolf \\ Department of Agricultural, Food, and Resource Economics, Michigan State University, East Lansing 48824
}

\begin{abstract}
Dairy herd reproductive performance and efficiency are closely tied to whole-farm profitability on commercial US dairy operations. Decision support assists farm managers seeking to determine economically optimal programs. Stochastic dominance is a risk efficiency criterion that was used to determine the preferred set of reproductive management programs. Stochastic dominance analysis of reproductive management programs, including visual heat detection without aids, Ovsynch, and Cosynch, was undertaken to facilitate management decisions regarding reproductive management programs. First-degree stochastic dominance identified the efficient set of reproductive management programs for those decision makers who simply prefer "more to less" or in this case prefer the higher value program. Seconddegree stochastic dominance was used to identify the efficient set for decision makers who prefer "more to less" at a diminishing rate, reflecting risk aversion. For each program, artificial insemination submission rate and conception rate were the outcome variables that involved risk. Ovsynch and Cosynch were found to be first- and second-degree dominant over visual heat detection in the example base case. Comparing Ovsynch and Cosynch revealed first- and second-degree stochastic dominance of Ovsynch over Cosynch. Managers with all risk preferences would prefer Ovsynch until an additional $9 \mathrm{~min}$ of labor were required for Ovsynch. Risk-averse managers would prefer Ovsynch to Cosynch until an additional 25 min of labor were required to breed with Ovsynch. This highlights that, in the example base case, risk-averse managers are willing to incur additional labor costs to breed with Ovsynch and avoid potential downside conception rate risks associated with Cosynch. Risk preferences of the manager affect which programs remain in the efficient set. Risk preferences of farm managers must be recognized when making reproductive program recommendations. Be-
\end{abstract}

Received June 2, 2008.

Accepted November 10, 2008.

${ }^{1}$ Corresponding author: olynknic@msu.edu cause dairy farmers are likely to be risk averse, seconddegree stochastic dominance is particularly important for their decisions.

Key words: stochastic dominance, reproductive management, decision support

\section{INTRODUCTION}

Dairy herd reproductive performance is integrally linked to profitability of commercial US dairy farms. Further, the direct link between reproductive performance and dairy herd management is widely accepted and has long been recognized in the dairy industry (Britt, 1985). In response to reproductive efficiency challenges, management programs have been developed to aid producers. Given the number of reproductive management programs available, assessments are necessary to determine the economically efficient programs for farm managers with given characteristics.

For many dairy farms the failure to efficiently detect estrus limits reproductive performance (Nebel and Jobst, 1998). Increased levels of production coupled with increased herd sizes have influenced how dairy farms manage dairy cattle reproduction (Pursley et al., 1997), spurring the development of programs to increase reproductive efficiency and performance. Today, many reproductive technologies are available for use on commercial dairy farms, including AI programs to synchronize estrus and ovulation, sex-sorted semen, computer-based record systems, and multiple aids for detection of estrus. Costs associated with reproductive management programs and with achieving a given level of reproductive performance vary across farms because of differing on-farm costs, facilities employed, previous levels of reproductive performance, management ability, and other economic factors.

Recent advances in reproductive management programs have focused on hormonal synchronization of dairy cattle. Synchronization programs can decrease expenses associated with estrus detection by shortening the time during which cattle must be observed through ovulation synchronization or by eliminating the need for estrus detection completely with timed AI (TAI). Timed ovulation allows for breeding cows by appoint- 
ment, thereby eliminating the need for heat detection, and has similar pregnancy outcomes to those obtained through AI performed after estrus detection (Pursley et al., 1997). Benefits for the dairy farm using systematic breeding programs include increased efficiency in the use of heat detection labor and the convenience of managing the herd under synchronization (Nebel and Jobst, 1998).

Farms may use more than one synchronization protocol. The program best suited to a particular operation depends on farm-specific costs, including costs of supplies and labor, manager's preferences, labor availability, and herd characteristics. In particular, potential distributions of outcomes associated with reproductive programs and the risk preferences of the farm manager aid in determining the farm-specific optimal program. Farm managers have heterogeneous risk preferences and may be classified as risk averse, risk neutral, or risk loving. The riskiness of a given program's outcome [i.e., the certainty with which a program will yield a given conception rate (CR) or AI submission rate (AISR)] affects the decision of a particular farm to adopt a given program. The primary objective of this study was to determine the economically optimal programs for dairy farmers while accounting for the risk involved in those reproductive management programs.

Several spreadsheet-based models (Groenendaal et al., 2004; Tenhagen et al., 2004; De Vries, 2006) have been developed to assess reproductive management decisions or costs associated with reproductive inefficiency. Groenendaal et al. (2004) created OptiCow to support economically optimal breeding and replacement decisions on dairy farms through the use of the marginal net revenue technique. Tenhagen et al. (2004) used field trials to compare reproductive efficiency and economic implications. The DairyVIP model by De Vries (2006) was used to estimate the value of a pregnancy. A commonly recognized theme in models that allow some flexibility in parameterization is that costs associated with supplies for reproductive programs and time required to administer the programs vary widely with managerial skill and experience levels. Further, differing gains in reproductive efficiency associated with a particular program underscore the need for sensitivity analyses. The models currently available do not explicitly consider risk associated with the outcomes of various programs or technologies, nor do they incorporate the risk attitudes of the decision maker.

Stochastic dominance is a risk efficiency criterion that has been employed in decision making in agriculture in various applications. Stochastic dominance is a powerful tool for identifying preferred or efficient sets of risky options for a decision maker with a given set of characteristics. Efficient sets are identified by com- paring the cumulative distribution function (CDF) of possible options with risky outcomes for the decision maker. Stochastic dominance analysis has been used in several areas of agricultural decision making, including preferred milking parlor investment options (Thomas et al., 1997), pest management (Zacharias and Grube, 1984), water-conserving irrigation strategies (Harris and Mapp, 1986), alfalfa management strategies on dairy farms (McGuckin, 1983), and tillage practices (Klemme, 1985).

This analysis assesses reproductive technology decisions under risk (i.e., uncertain outcomes of AISR and $\mathrm{CR}$ programs) to accomplish the main objective of determining the economically optimal programs for dairy farms with various characteristics. Stochastic dominance was used to separate the sets of risky options and identify efficient sets for decision makers given risk preferences. Through first-degree stochastic dominance (FSD) the efficient set of reproductive management programs for all those decision makers who simply prefer the higher value program can be identified. The use of second-degree stochastic dominance (SSD) analysis allows identification of the efficient set of reproductive management programs for decision makers who prefer "more to less" at a diminishing rate, reflecting risk aversion. Analysis of the sensitivity of results to changes in key on-farm parameters was used to assess differences in efficient sets for farms with varying cost structures. Given that dairy farmers have heterogeneous risk preferences, efficient sets of programs were sought for broad risk preference groups of farm managers.

\section{MATERIALS AND METHODS}

The variability of responses to a specific program and the attitude of the decision maker toward that variability were assessed through stochastic dominance analysis. By including the risk associated with the response to the program, as well as the willingness to accept risk by the decision maker, the problem for the farm manager becomes maximizing expected utility. The inclusion of risk associated with the reproductive performance resulting from a specific program or technology and the risk attitude of the decision maker add an important component to existing models and decision support tools. Farm-specific costs for labor and program administration aid in determining which programs or protocols are stochastically dominant for farms with a given set of characteristics. The risk attitudes (i.e., whether the manager is risk averse, risk neutral, or risk loving) were expected to be important in determining which reproductive programs are adopted by particular farm managers. 


\section{Stochastic Budget Development}

A series of budgets was developed in Excel (Microsoft, Redmond, WA) to simulate reproductive performance under different reproductive management programs. The amount of labor required to administer injections is an important component of reproductive program costs and is a function of farm facilities and employee skill level. Similarly, visual heat detection program costs vary depending on hourly labor costs and the efficiency with which heats are detected. Reproductive program costs were calculated on a per-cow basis to facilitate comparison across programs. Heat detection program costs were adjusted to obtain a per-cow basis by dividing the cost of heat detection for a group of cows over the number of cows in that group.

The reproductive management programs chosen for use in this analysis were Ovsynch, Cosynch, and visual heat detection without the use of aids. Ovsynch consists of 1) an injection of $\mathrm{GnRH}, 2)$ an injection of $\mathrm{PGF}_{2 \alpha} 7$ d later, 3) a second GnRH injection $48 \mathrm{~h}$ later, and 4) breeding $24 \mathrm{~h}$ after the second GnRH injection (Pursley et al., 1995). To resynchronize those cows not conceiving to the first AI, all cows were assumed to receive a GnRH injection at a nonpregnant diagnosis beginning $26 \mathrm{~d}$ after Ovsynch TAI was performed. Cosynch differs from Ovsynch only in the timing of the TAI. Unlike in Ovsynch, cows in the Cosynch program are bred concurrently with the second GnRH injection, thereby eliminating the need for an additional cow handling.

To compare values across programs, the values must be assessed subject to achieving a specified level of reproductive success; in this analysis, the programs were assessed subject to cows being bred for 6 AI (Olynk and Wolf, 2008b).

Heat detection rate is the percentage of eligible cows correctly detected in estrus in a 21-d period (Plourd, 2000), whereas AISR is the percentage detected in estrus and bred in that same period. The CR was assumed to decrease as AI number increased. The second and third or later AI CR were assumed to be 90.7 and 81.4\%, respectively, of the first AI CR, based on Cassell (2001). The AISR was held constant for each program.

To calculate the expected value of a reproductive program the predetermined cutoff of 6 AI was achieved and the cow value was assessed by incorporating her retention pay-off (RPO). The RPO is the difference in total net returns from keeping the cow in the herd versus culling and replacing her immediately. The RPO is defined as the total additional profit expected from attempting to keep the cow until her optimal age compared with immediately removing her from the herd and replacing her (taking into account changes in involuntary culling); the higher the RPO, the more valuable the animal and, thus, the larger the loss if the cow is culled at that time (Groenendaal et al., 2004). DairyVIP version 1.1 (De Vries, 2006) was used to obtain the RPO values. Input values used to obtain the $\mathrm{RPO}$ for pregnant and nonpregnant cows were from De Vries (2006) unless otherwise specified below.

During each period, the RPO of a cow that conceived in that current period was multiplied by the CR for that period to obtain the RPO value of a pregnant cow. The probability that the cow remained open after the last breeding period was multiplied by the RPO of an open cow in the period in which the cut-off criterion was reached. The cost per breeding in calculating the RPO was set at zero because breeding costs were accounted for in calculating the value of the breeding programs. Included in the model were RPO values greater than zero; values that were negative were evaluated at zero. A charge for feed and yardage (assumed at $\$ 2 / \mathrm{d}$ ) was used in calculating the breeding program cost for each period that the cow remained nonpregnant after her first AI. Cows were assumed to be submitted to breeding beginning at their third month in lactation and were bred once every eligible period until a pregnancy occurred or the cutoff criterion was reached. Eligible periods for breeding differed depending upon whether cows were assigned for breeding by visual heat detection or by a synchronization program. Cows bred to visual heat detection were assumed to have breeding periods of $21 \mathrm{~d}$. For synchronization programs, cows were assumed to start resynchronization upon a nonpregnant diagnosis at $26 \mathrm{~d}$ after TAI.

Heat detection program costs, assessed on a perperiod per-cow basis were calculated as

$$
\begin{gathered}
\mathrm{PROG}_{\mathrm{HD}}=(\{[(\mathrm{TIME} \times \mathrm{OBS}) \times \mathrm{PER}] \\
\left.\left.\times \mathrm{LABOR}_{\mathrm{HD}}\right\} / \mathrm{COWS}\right)+\mathrm{AID}+(\mathrm{CI} \times \mathrm{AISR}),
\end{gathered}
$$

where $\mathrm{PROG}_{\mathrm{HD}}=$ heat detection program costs per cow per period; TIME $=$ heat detection minutes per observation for a group of cows; OBS = number of times the group is observed per day; PER = number of days in a single breeding period; $\mathrm{LABOR}_{\mathrm{HD}}=$ cost of labor $(\$ / \mathrm{min})$ to perform heat detection; COWS $=$ number of cows in the group; AID = per period cost $(\$)$ per cow of heat detection aid utilized, $\mathrm{CI}=\operatorname{cost}(\$)$ of an artificial insemination, and AISR $=$ AI submission rate.

Synchronization program costs, assessed on a perperiod per-cow basis were calculated as

$$
\begin{gathered}
\text { PROG }_{\text {Synch }}=\left(\mathrm{P}_{\mathrm{GnRH}} \times \mathrm{X}_{\mathrm{GnRH}}\right)+\left(\mathrm{P}_{\mathrm{PGF} 2 \alpha} \times \mathrm{X}_{\mathrm{PGF} 2 \alpha}\right) \\
+\left(\mathrm{MIN} \times \mathrm{INJ} \times \text { Labor }_{\mathrm{Inj}}\right)+(\mathrm{CI} \times \mathrm{AISR}),
\end{gathered}
$$


where $\mathrm{PROG}_{\text {Synch }}=$ synchronization program costs per cow per breeding period; $\mathrm{P}_{\mathrm{GnRH}}=$ cost of $\mathrm{GnRH}$ per injection; $\mathrm{X}_{\mathrm{GnRH}}=$ number of $\mathrm{GnRH}$ injections administered; $\mathrm{P}_{\mathrm{PGF} 2 \alpha}=$ cost of $\mathrm{PGF}_{2 \alpha}$ per injection; $\mathrm{X}_{\mathrm{PGF} 2 \alpha}$ $=$ number of $\mathrm{PGF}_{2 \alpha}$ injections administered; $\mathrm{MIN}=$ minutes to give a single injection; INJ $=$ total number of injections in the series; $\mathrm{LABOR}_{\mathrm{Inj}}=$ cost of labor $(\$ / \mathrm{min})$ to give injections; $\mathrm{CI}=$ cost of an artificial insemination; and AISR $=$ AI submission rate.

In calculating the program costs for both synchronization and visual heat detection programs, the program labor costs were assessed as wages paid if the task was performed by paid labor or as opportunity cost of the labor if unpaid labor was used. Opportunity cost is the cost of having that labor participate in the reproductive management tasks rather than in the next best alternative activity. Because many herds, especially smaller herds, use unpaid family or managerial labor for their reproductive management program, a charge for the opportunity cost of unpaid labor must be included to assess the true costs associated with various reproductive management programs. In many cases, such as when managers perform heat detection, the opportunity cost for unpaid labor was more expensive than hired labor.

The formula used to calculate the expected value of each program, with $\mathrm{NCF}_{\mathrm{T}}$ for the last time period including the RPO values:

$$
\begin{gathered}
\mathrm{NCF}_{\mathrm{t}}=\left(\mathrm{CR}_{\mathrm{t}} \times \mathrm{RPOPREG}_{\mathrm{t}}\right)-\left[\left(1-\mathrm{PR}_{\mathrm{cum}}\right)\right. \\
\times \text { Feed }]-\mathrm{PROG}_{\mathrm{j}} \text { for } t=0, \ldots, \mathrm{T}-1
\end{gathered}
$$

and

$$
\begin{gathered}
\mathrm{NCF}_{\mathrm{T}}=\left(\mathrm{CR}_{\mathrm{T}} \times \mathrm{RPOPREG}_{\mathrm{T}}\right)-\left[\left(1-\mathrm{PR}_{\mathrm{cum}}\right)\right. \\
\times \text { Feed }]-\mathrm{PROG}_{\mathrm{j}}+\left[\left(1-\mathrm{PR}_{\mathrm{cum}}\right)\right. \\
\left.\times \mathrm{RPOOPEN}_{\mathrm{T}}\right] \text { for } t=\mathrm{T}
\end{gathered}
$$

where $\mathrm{NCF}_{\mathrm{t}}=$ net cash flow in period $t$; PROG $=$ Program costs for either synchronization or heat detection program per cow per period; $\mathrm{CR}_{\mathrm{t}}=$ current $\mathrm{AI} \mathrm{CR}$; $\mathrm{PR}_{\text {cum }}=$ cumulative probability of pregnancy, defined as sum of PR from 0 through the current AI, in time $t$, (e.g., $\mathrm{PR}_{\text {cum }, 3}=\mathrm{CR}_{0}+\left(1-\mathrm{CR}_{0}\right) \times \mathrm{CR}_{1}+\left(1-\mathrm{CR}_{0}\right)$ $\left.\times\left(1-\mathrm{CR}_{1}\right) \times \mathrm{CR}_{2}\right) ;$ Feed $=$ per-period feed and yardage cost of nonpregnant cow $=(\$ 2.00$ per day $\times$ days per period); $\mathrm{PROG}_{\mathrm{j}}=$ cost of program $j$, where $j$ is the reproductive program used on the cow; $\mathrm{RPOOPEN}_{\mathrm{T}}=$ $\mathrm{RPO}$ at time $\mathrm{T}$ for an open cow; and RPOPREG $\mathrm{T}_{\mathrm{T}}=$ $\mathrm{RPO}$ at time $\mathrm{T}$ for a pregnant cow.
The value of the strategy was the sum of the values from time period 0 through $\mathrm{T}$, in which the final time period, $\mathrm{T}$, was set at the predetermined cutoff criteria of $6 \mathrm{AI}$ :

$$
\text { Program value }=\sum_{t=0}^{T}\left[N C F_{t}\right] \text {, }
$$

where $\mathrm{NCF}_{\mathrm{t}}=$ net cash flow in period $t$.

Stochastic variables were incorporated into the budgets to account for the riskiness of the AISR and $\mathrm{CR}$ in response to various reproductive management programs. Rather than the inclusion of a single value or a deterministic variable, stochastic variables allow the AISR and CR to be assessed over a distribution of outcomes. Multiple draws, or iterations, from these distributions for AISR and CR can then be taken to allow simulation of how a program may perform. Simulation models that do not account for the riskiness of outcomes give a deterministic result in response to the input variables. Stochastic models are used to account for variables for which the values are not known with certainty, although for which a known probability distribution exists. Through the use of stochastic variables, the interactions of risky variables (in particular the interaction of the CR and AISR to achieve a given PR) allow simulation of how a program may perform under alternative outcomes. Stochastic dominance is a popular method for ranking risky alternatives without in-depth knowledge about the preferences of the decision maker (McCarl, 1990).

\section{Stochastic Dominance Analysis}

Stochastic dominance is a powerful risk efficiency criterion. Such criteria separate a set of possible actions into an efficient set that is preferred by the decision maker and a risk-inefficient set that is undesirable to the decision maker (Thomas et al., 1997). Efficient sets are identified by comparing the CDF of possible options for the decision maker that have risky outcomes. Efficient sets are defined to include the choice of every decision maker (farm manager) to whom the specified decision rules apply. Efficient sets for both FSD and SSD were evaluated in this analysis. An underlying assumption for both of FSD and SSD is that the alternatives compared, in this case the reproductive management programs, are mutually exclusive, meaning that one program or the other must be chosen and that a program that combines the 2 options is not feasible.

According to Mas-Collel et al. (1995) FSD has the intuitive meaning that "the distribution $\mathrm{F}($.) yields un- 
ambiguously greater returns than the distribution $\mathrm{G}($.$) "$ (p. 195). The distribution $\mathrm{F}$ (.) first-order stochastically dominates $\mathrm{G}($.$) if, for every nondecreasing function u:$ $\int u(x) d F(x) \geq \int u(x) d G(x)$, where $\mathrm{u}$ indicates the utility function of the decision maker (Mas-Collel et al., 1995). Therefore, FSD can identify the efficient set for all those decision makers who prefer "more to less" or, in this case, prefer the higher value program. The SSD analysis then seeks to introduce a comparison based on relative riskiness or dispersion. The efficient set for decision makers who prefer "more to less" at a diminishing rate, reflecting risk aversion, was determined through the use of SSD. The risk aversion of the decision maker is evident in the fact that the utility function is a nondecreasing concave function. For any 2 distributions $\mathrm{F}($.$) and \mathrm{G}(),. \mathrm{F}($.) second-order stochastically dominates $\mathrm{G}($.$) if for every nondecreasing concave function$ $\mathrm{u}: \int u(x) d F(x) \geq \int u(x) d G(x)$, where $\mathrm{u}$ indicates the utility function of the decision maker (Mas-Collel et al., 1995). Notice that in FSD the utility function of the decision maker was only constrained to be a nondecreasing function, whereas in SSD the utility function is nondecreasing and also concave. Second-degree stochastic dominance analysis does not require identical means, although the assumption of identical means reduces the analysis to assessing one distribution as a mean-preserving spread of the other. A risk-averse individual prefers a mean-preserving contraction over a meanpreserving spread. Through the use of these rules, efficient sets were identified for those decision makers fulfilling the aforementioned assumptions.

An economic analysis was conducted to determine the optimal reproductive program for farms with a given set of characteristics. Stochastic budgeting was used to account for the uncertainties in the decision, namely the AISR and CR resulting from a reproductive program, and to give an indication of the distribution of the outcomes expected. This analysis began by running the decision tool for multiple iterations with key parameters (i.e., CR, AISR, and the resulting PR) distributed across the range of values identified in previous studies; namely Olynk and Wolf (2008a), Stevenson et al. (2008), and J. R. Pursley (Michigan State University, East Lansing; personal communication). In this way, data from previous reproductive performance studies were used in conjunction with survey data to parameterize the economic analysis. The stochastically efficient or efficient set was then identified by comparing the CDF of the risky reproductive program alternatives. Through the use of @RISK Version 4.5 (Palisade Corp., Newfield, NY) the budget for each program was evaluated for 10,000 iterations. The resulting CDF of the risky alternatives were graphed for ease of visual observation in Stata software (Intercooled Stata for Windows, version 8.2, Stata Corporation, College Station, TX).

Key reproductive management program parameters were made stochastic. In particular, farm-size differences in program costs were assessed to highlight sensitivity to farm-specific costs. Triangular distributions were used to parameterize the AISR and CR associated with the reproductive management programs. Often triangular distributions are used in such cases with limited sample data because only minimum, maximum, and most likely values are necessary to parameterize the model. Multiple scenarios are necessary to identify the efficient sets for decision makers with various baseline farm characteristics. A general base-case example scenario was constructed for illustration from previous survey data under the assumptions that on-farm labor costs $\$ 12.78 / \mathrm{h}$ for both heat detection and giving injections, costs per AI were $\$ 17.30,2.15 \mathrm{~h} / \mathrm{d}$ were devoted if visual heat detection was used for a group of 100 cows being observed at a single time, labor time to give a single injection was $2.10 \mathrm{~min}$ if synchronization was used, GnRH cost $\$ 3.59$ per dose, and $\mathrm{PGF}_{2 \alpha}$ cost $\$ 2.52$ per dose (Olynk and Wolf, 2008a).

Visual heat detection without aids, Ovsynch, and Cosynch were investigated because the Ovsynch program protocol is widely used in the industry and the protocols for Ovsynch and Cosynch are nearly identical, varying only by timing of AI. Cosynch is essentially a specific modification of Ovsynch in which cows receive TAI concurrently with the second GnRH injection. Cosynch has the advantage of allowing dairy farms to sort and restrain cows one less time than the Ovsynch program, and allows for all cow handling to occur at the same time daily (Fricke, 2003). Although there may be advantages for the Cosynch program from a cattle handling standpoint, optimal conception rates are not achieved using Cosynch (Pursley et al., 1998). Because of the similarities between the Cosynch and Ovsynch programs, they offer an interesting comparison, because dairy farms considering one of these programs would also likely consider the other.

The distribution used for the CR to visual heat detection was parameterized using survey data using a triangular distribution with a minimum of $26 \%$, maximum of $60 \%$, and most likely value of $41 \%$ (Olynk and Wolf, 2008a). For Ovsynch, the CR was also distributed triangularly, although with a minimum of $23 \%$, maximum of $57 \%$, and a most likely value of $38 \%$. The minimum and maximum values were adjusted slightly from the CR distribution used for visual heat detection, as mixed opinions exist regarding the differences in CR between AI to visually detected heat versus TAI. The 
most likely value for Ovsynch was taken from Pursley et al. (1997). The triangular distribution for CR under Cosynch reflected the lower CR expected with Cosynch versus Ovsynch, which is expected to be approximately 6 percentage points, on average (J. R. Pursley, Michigan State University, East Lansing; personal communication). The CR distribution included a minimum of $21 \%$, a maximum of $55 \%$, and a most likely value of $32 \%$.

The triangular distribution used for visual heat detection without aids was parameterized using survey data and had a minimum of $20 \%$, maximum of $75 \%$, and a most likely of $52 \%$ (Olynk and Wolf, 2008a). The AISR for TAI used for Ovsynch was a triangular distribution of 96,100 , and $99 \%$ for the minimum, maximum, and most likely values, respectively, because most cows were bred to TAI; similarly, values for Cosynch were 97, 100, and $99 \%$ because cows were almost certainly bred TAI, except under unexpected circumstances. The $\mathrm{PR}$ resulting from each of these programs was affected by the distributions of the AISR and CR under each program.

Numerous base scenarios are possible to allow stochastic dominance assessments for farms of various herd-sizes. Multiple iterations must be run for farms with a given herd-size and on-farm labor cost. By holding these factors describing the general characteristics of the farm fixed and running several iterations with the AISR and CR being treated as stochastic, CDF can be generated and compared to determine efficient sets for decision makers with different risk preferences and given certain farm characteristics.

Farms vary on whether they incur additional costs from breeding cows with Ovsynch. Farms may have facilities that enable automatic sorting, thereby making an additional cow-handling efficient and inexpensive. Other farms, however, may incur significant costs associated with sorting and handling cattle an additional time for breeding with Ovsynch versus Cosynch. Such differences warrant sensitivity analysis. A comparison case was constructed for sensitivity analysis where additional costs were incurred for breeding with Ovsynch versus the other programs. There is an inherent tradeoff between these programs in that cattle must be handled an additional time with Ovsynch for breeding, but the expected CR is slightly higher than that with Cosynch. To account for the additional handling associated with breeding in the Ovsynch program, the cost per AI was adjusted from the $\$ 17.30$ described above to include 15 additional min of labor cost, which was assessed at the $\$ 12.78 / \mathrm{h}$ as described above. Therefore, the cost per AI for the Ovsynch protocol were assessed at $\$ 20.50$ to include the $\$ 17.30$ per AI from the base scenario plus $\$ 3.20$ in added labor costs for an additional cattle handling. All other costs here held constant with the base example scenario described above.

To assess the sensitivity of the analysis to farmspecific costs, sensitivity analysis was conducted using the same reproductive programs as above. In particular, sensitivity to on-farm costs and cow group sizes were assessed. Such sensitivity analysis highlights the differences that the initial farm costs for AI services and program supplies and group size assumptions can have on the analysis. Farms with $<100$ cows often have greater costs for purchasing hormones and greater per AI costs. For comparison, a scenario indicative of smallfarm costs and values was constructed from survey data. Parameterization of the small farm comparison used the following parameters: labor costs were maintained at $\$ 12.78 / \mathrm{h}$, per AI costs were $\$ 21.00$ for visual heat detection and Cosynch, per AI cost was $\$ 24.20$ for Ovsynch and was adjusted in the same way as before, $2.67 \mathrm{~h} / \mathrm{d}$ were devoted to heat detection, labor time to give a single injection was $1.7 \mathrm{~min}, \$ 4.49$ per dose of GnRH and $\$ 3.10$ per dose of $\mathrm{PGF}_{2 \alpha}$. To adjust for the smaller total farm size, the number of cows observed at a single time was assumed to be 35 .

\section{RESULTS AND DISCUSSION}

Farm-specific technology adoption decisions were found to be sensitive to on-farm costs and individual farm characteristics. Examining the uncertainty of response in performance to reproductive management programs, and therefore changes in costs associated with reproductive management programs, assisted in determining why farms select reproductive management programs to maximize profitability through reproductive management. In this way, risk preferences affect which technologies or programs are in the efficient set.

The descriptive statistics of the PR under each reproductive program are displayed in Table 1 . The PR resulting from each of these programs was affected by the distributions of the AISR and CR under each program. Table 2 illustrates the summary statistics for the values of the programs resulting from the 10,000 iterations run on the base example analysis. In comparing mean values for programs, Ovsynch had the highest mean value, Cosynch had the second highest mean value, and visual heat detection had the lowest mean program value. The standard deviation was lowest for the Ovsynch program, second lowest for the Cosynch program, and highest for visual heat detection. Therefore, if a program were selected simply by highest mean and lowest standard deviation, the Ovsynch program would be preferred to the Cosynch program and visual heat detection programs, which had lower means and higher standard deviations than the Ovsynch program. 
Figure 1 illustrates the CDF of the various reproductive management programs available. In Figure 1, both Ovsynch and Cosynch are FSD and SSD over visual heat detection without aids. When comparing Ovsynch and Cosynch to one another, there is FSD and SSD of Ovsynch over Cosynch. Note that all FSD and SSD outcomes described above are subject to the parameters outlined in this example. For this example case, the efficient set for FSD includes only Ovsynch because it is FSD compared with visual heat detection and Cosynch. Additionally, Ovsynch was the efficient set for SSD because it is SSD over visual heat detection and Cosynch. The result that Ovsynch was preferred to Cosynch supports a common perception in the industry that producers prefer to handle cows an additional time, as with Ovsynch, rather than risk a lower CR, as with Cosynch. Managers of all risk preferences were found to prefer Ovsynch when the additional handling of cows for breeding does not yield additional costs. To assess a more realistic situation regarding the additional costs of breeding with Ovsynch, additional labor costs were added to determine efficient sets under differing labor requirements.

Breakeven analyses were conducted to determine at which minimum and maximum values in the distributions for CR and AISR the efficient sets of programs changed. The CR for visual heat detection was evaluated to determine at what maximum value of $\mathrm{CR}$ in the distribution the efficient sets of programs would be altered. If the maximum value for the $\mathrm{CR}$ in the visual heat detection program is $>60 \%$, then Cosynch was no longer FSD over visual heat detection. If the maximum value for the $\mathrm{CR}$ in the visual heat detection program is $>64 \%$, then Ovsynch was no longer FSD over visual heat detection. A maximum value for the triangularly distributed $\mathrm{CR}$ in the distribution could not be identified in the example scenario at which Ovsynch and Cosynch no longer was SSD over visual heat detection. In other words, even if the maximum value for the CR in the triangular distribution were set to $100 \%$, Ovsynch and Cosynch were still SSD over visual heat detection.

The triangular distribution for AISR was also assessed to determine at what maximum value for AISR in the distribution the efficient set would be altered. If the maximum value for the AISR in the visual heat detection program is above $75 \%$, then Cosynch would no longer be FSD over visual heat detection. If the maximum value for the AISR in the visual heat detection program is $>80 \%$, then Ovsynch would no longer be FSD over visual heat detection. A maximum value for the triangularly distributed AISR in the distribution could not be identified in the example scenario in which Ovsynch and Cosynch were no longer SSD over visual heat detection. In other words, even if the maximum
Table 1. Summary statistics for pregnancy rates in example analysis

\begin{tabular}{lccc}
\hline \multirow{3}{*}{ Statistic } & \multicolumn{3}{c}{ Pregnancy rate } \\
\cline { 2 - 4 } Mean & Visual heat detection & Ovsynch & Cosynch \\
Median & 0.208 & 0.386 & 0.356 \\
Mode & 0.205 & 0.383 & 0.348 \\
SD & 0.261 & 0.425 & 0.353 \\
Minimum & 0.059 & 0.068 & 0.070 \\
Maximum & 0.064 & 0.227 & 0.208 \\
& 0.415 & 0.564 & 0.541 \\
\hline
\end{tabular}

${ }^{1}$ Summary statistics are presented for the pregnancy rates calculated for the visual heat detection, Ovsynch, and Cosynch programs for the example analysis of 10,000 iterations.

value for the AISR in the triangular distribution was set to 100\%, Ovsynch and Cosynch were still SSD over visual heat detection.

Breakeven values for AISR and CR that would alter the dominance between Ovsynch and Cosynch were determined. The distribution for the CR outcome for Cosynch was altered by changing the minimum value to $23 \%$, maximum value to $57 \%$, most likely value to $38 \%$, and changing both the minimum to $23 \%$ and maximum to $57 \%$ at the same time to provide comparisons to the distribution of $\mathrm{CR}$ outcomes with the Ovsynch program. None of these alternatives altered the dominance between Ovsynch and Cosynch. Looking at Ovsynch, the AISR for Ovsynch was altered by reducing the minimum value for the AISR in the distribution. If the minimum value for the AISR for Ovsynch is below $77 \%$, Ovsynch is no longer FSD or SSD over Cosynch.

Figure 2 displays the CDF comparison when Ovsynch incurs an additional breeding cost of $\$ 3.20$, or an additional 15 min of labor costs per AI compared with the other 2 programs. Both Ovsynch and Cosynch are FSD (and SSD) over visual heat detection in this case. The interesting comparison is between Ovsynch and Cosynch. Note that neither Ovsynch nor Cosynch was FSD when 15 additional min of labor are required for the Ovsynch program. When assessing SSD, Ovsynch

Table 2. Summary statistics for program values in example analysis ${ }^{1}$

\begin{tabular}{lccc}
\hline & \multicolumn{3}{c}{ Program values (\$ per program per year) } \\
\cline { 2 - 4 } Statistic & Visual heat detection & Ovsynch & Cosynch \\
\hline Mean & 490.54 & 593.92 & 562.92 \\
Median & 494.86 & 599.13 & 564.78 \\
Mode & 533.58 & 556.16 & 560.41 \\
SD & 76.43 & 53.42 & 60.40 \\
Minimum & 248.42 & 431.68 & 396.89 \\
Maximum & 680.71 & 696.41 & 683.93 \\
\hline
\end{tabular}

${ }^{1}$ Program values were computed as explained in the text. Summary statistics for program values for the visual heat detection, Ovsynch, and Cosynch programs are provided. 


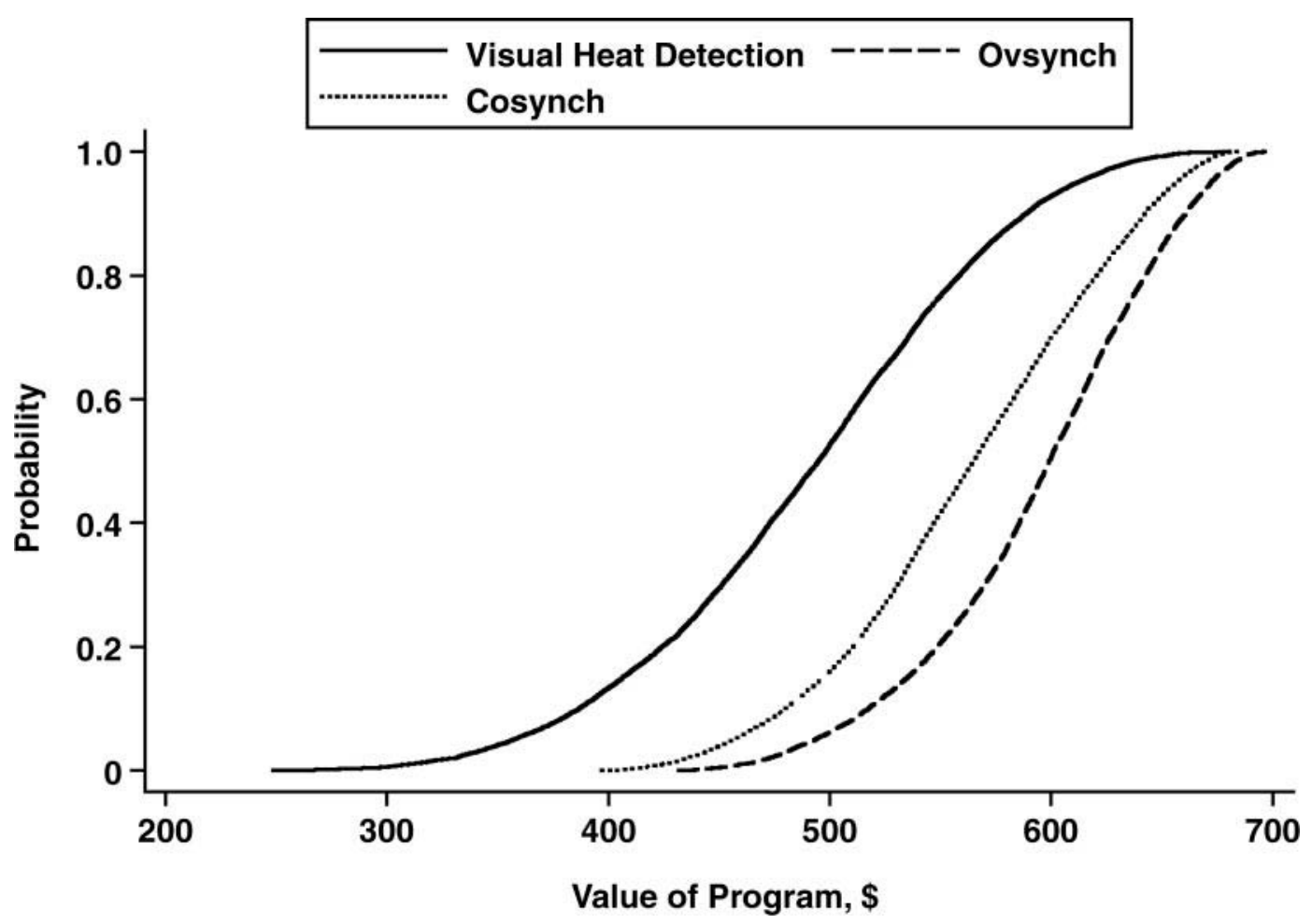

Figure 1. Cumulative density functions for example analysis (of 10,000 iterations) comparing Ovsynch, Cosynch, and visual heat detection programs.

was SSD over Cosynch. The additional 15 min of labor costs, totaling $\$ 3.20$, incurred per AI in the Ovsynch program in this case did not increase the cost sufficiently to alter the SSD of Ovsynch over Cosynch. The SSD of Ovsynch over Cosynch indicated that riskaverse managers still preferred the Ovsynch program to the Cosynch program, even when they have to incur additional costs associated with handling cows an additional time, an assumed 15 min in this example, for breeding purposes in Ovsynch. Given this analysis, in the case in which Ovsynch incurs 15 min of additional labor costs over the other 2 programs, the efficient set for FSD was Ovsynch and Cosynch because both dominate visual heat detection. The efficient set for SSD was Ovsynch because it dominates visual heat detection and the Cosynch program. A common perception within the dairy industry regarding the use of Ovsynch to achieve the benefits of a synchronization program with as little depression of the CR as possible was supported by the efficient sets identified in this analysis. Risk-averse managers were found to prefer the Ovsynch program over the Cosynch program, highlighting the preference for additional time spent handling cows versus decreased CR, as would be expected given the popularity of Ovsynch in the industry and the hypothesized risk aversion of dairy producers. A breakeven analysis was conducted to determine the number of minutes for an additional cow-handling at which Ovsynch no longer was FSD over Cosynch. If an additional cow-handling requires 9 min or more of additional labor but less than 25 additional min, Ovsynch was no longer FSD over Cosynch, but Ovsynch was still SSD over Cosynch. If 25 min or more of additional labor are required for an additional cow-handling, no SSD (or FSD) exists between Ovsynch and Cosynch.

Farm location, farm size, management, and production technology sets affect the farm costs associated with reproductive management programs. To assess sensitivity to farm costs, FSD and SSD was assessed for small farms, as indicated in the Materials and Methods section. Sensitivity to farm costs can be seen in the higher costs associated with reproductive programs on small farms, thereby resulting in smaller total values in this analysis. Perhaps most significant is the extreme difference in the visual heat detection without aids program. Costs are considerably higher for small farms because of watching fewer cattle at a time for visual heat detection and greater per-injection hormone costs for synchronization. In addition, per-AI costs were higher on small farms. For small farms ( $<100$ cows) the efficient set for FSD was Ovsynch and Cosynch because both FSD over visual heat detection. Ovsynch 


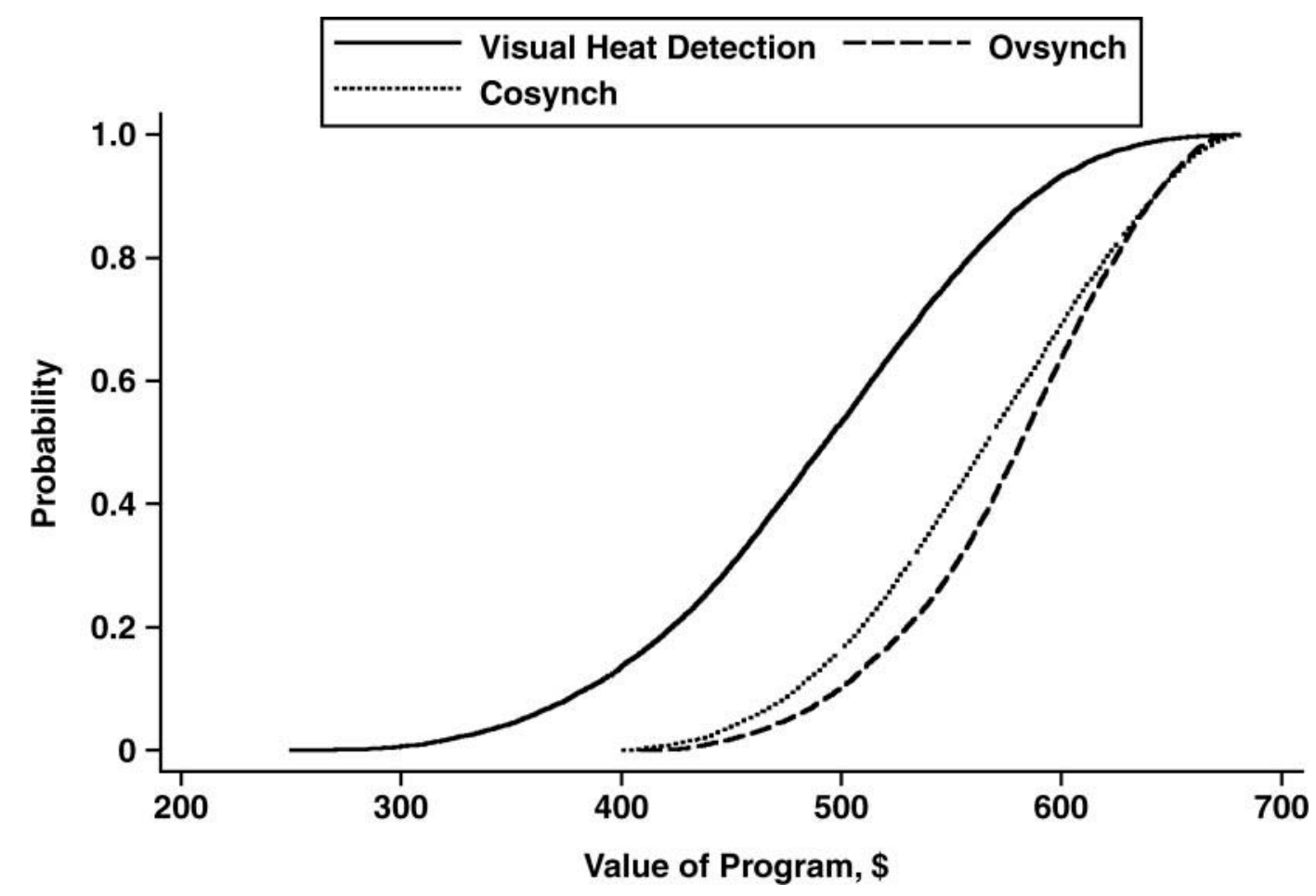

Figure 2. Sensitivity analysis in example analysis (of 10,000 iterations) of Ovsynch, Cosynch, and visual heat detection with Ovsynch incurring 15 min of additional breeding cost.

was SSD over both the Cosynch and visual heat detection programs.

Through FSD and SSD analysis, the efficient sets of reproductive programs have been identified for those producers who 1) prefer a higher value program (FSD) and 2) prefer a higher value program and are risk averse (SSD). It is important to note that the programs that are present in the efficient set depend on the parameters set up in the example problem. Those programs in the efficient set will be different for farms with varying on-farm labor costs, labor efficiencies, and decision-maker preferences. This fact strengthens the notion that regional data or farm-size-specific data sets would be useful in parameterization of this analysis to allow costs that are more specific to a given locale.

\section{CONCLUSIONS}

Dairy herd reproductive performance is closely tied to whole-farm profitability for commercial US dairy operations. Identification of optimal programs for farms with a given set of general characteristics (i.e., operator risk attitudes, farm size, and on-farm costs) is necessary to enable recommendations to dairy farm operators and to gain further understanding of why farms adopt the reproductive technologies that they do. In this analysis stochastic variables were incorporated into a series of budgets to account for the riskiness of the reproductive program outcomes, namely AISR and CR. To determine the efficient sets of reproductive programs for decision makers with heterogeneous risk preferences FSD and SSD were used. This analysis has highlighted that risk aversion affects which programs remain in the efficient set, and the SSD analysis is particularly important for the dairy industry because dairy farmers are likely risk averse.

Perhaps the most interesting finding, highlighted through the base example case, was the FSD and SSD of Ovsynch over Cosynch, which indicates that decision makers of all risk preferences prefer Ovsynch over Cosynch. This dominance of Ovsynch indicates that decision makers do not want to take the CR risks associated with Cosynch. Although the synchronization programs employ the same number and type of hormonal injections, the timing of these injections affects the CR. Managers of all risk preferences would prefer Ovsynch until 9 additional min of labor were required for Ovsynch, whereas risk-averse managers would prefer Ovsynch to Cosynch until an additional 25 min of labor were required to breed with Ovsynch. This SSD analysis highlights that risk-averse managers are willing to incur additional labor costs to experience the CR distribution associated with Ovsynch rather than accept the CR distribution of Cosynch. 
Given the flexibility of the on-farm decision tool, parameterization of the model is possible for regional or even farm-specific data. When assessing only small farms, all program costs were found to be greater than the general assessment, indicating that regions with large proportions of small farms will find such analysis particularly important in assessing the optimal program for their operations. Further, farm costs such as labor costs associated with breeding for a particular program are important in identifying efficient sets for farms with given characteristics. Overall, the incorporation of the risk preferences of the decision maker is an important contribution to farm-level decision making. By identifying efficient sets of programs for decision makers with various risk preferences we are better able to make recommendations for managers with given farm characteristics.

\section{REFERENCES}

Britt, J. H. 1985. Enhanced reproduction and its economic implications. J. Dairy Sci. 68:1585-1592.

Cassell, B. 2001. Subject: Service sires for problem breeders. Virginia Cooperative Extension. Dairy Pipeline. http://www.ext.vt.edu/ news/periodicals/dairy/2001-07/servicesires.html Accessed March $25,2008$.

De Vries, A. 2006. Economic value of a pregnancy in dairy cattle. J. Dairy Sci. 89:3876-3885.

Fricke, P. M. 2003. Monitoring reproduction from the starting gate. Pages 77-88 in Proc. 6th Western Dairy Management Conference; http://www.wdmc.org/proceed.htm Accessed Sept. 7, 2008.

Groenendaal, H., D. T. Galligan, and H. A. Mulder. 2004. An economic spreadsheet model to determine optimal breeding and replacement decisions for dairy cattle. J. Dairy Sci. 87:2146-2157.

Harris, T. R., and H. P. Mapp. 1986. A stochastic dominance comparison of water-conserving irrigation strategies. Am. J. Agric. Econ. 68:298-305.

Klemme, R. M. 1985. A stochastic dominance comparison of reduced tillage systems in corn and soybean production under risk. Am. J. Agric. Econ. 67:550-557.

Mas-Collel, A., M. D. Whinston, and J. R. Green. 1995. Microeconomic Theory. Oxford University Press Inc, Oxford, UK.
McCarl, C. A. 1990. Generalized stochastic dominance: An empirical examination. Southern J. Agric. Econ. 22:49-56.

McGuckin, T. 1983. Alfalfa management strategies for a Wisconsin dairy farm: An application of stochastic dominance. N. Cent. J. Agric. Econ. 5:43-49.

Nebel, R. L., and S. M. Jobst. 1998. Symposium: Gonadotropinreleasing hormone and prostaglandin for estrus detection. J. Dairy Sci. 81:1169-1174.

Olynk, N., and C. Wolf. 2008a. A Survey of Reproductive Management Strategies on US Commercial Dairy Farms. Staff Paper 2008-02. Agricultural, Food, and Resource Economics, Michigan State University, East Lansing.

Olynk, N. J., and C. Wolf. 2008b. Economic analysis of reproductive management strategies on us commercial dairy farms. J. Dairy Sci. 91:4082-4091.

Plourd, R. 2000. Five repro measures you can use. Dairy Herd Management. http://www.dairyrepro.com/LI/five.htm Accessed on Sep. 12, 2008.

Pursley, J. R., M. R. Kosorok, and M. C. Wiltbank. 1997. Reproductive management of lactating dairy cows using synchronization of ovulation. J. Dairy Sci. 80:301-306.

Pursley, J. R., M. O. Mee, and M. C. Wiltbank. 1995. Synchronization of ovulation in dairy cows using $\mathrm{PGF}_{2 \alpha}$ and $\mathrm{GnRH}$. Theriogenology 44:915-923.

Pursley, J. R., R. W. Silcox, and M. C. Wiltbank. 1998. Effect of time of artificial insemination on pregnancy rates, calving rates, pregnancy loss, and gender ratio after synchronization of ovulation in lactating dairy cows. J. Dairy Sci. 81:2139-2144.

Stevenson, J. R., D. E. Tenhouse, R. L. Krisher, G. C. Lamb, J. E. Larson, C. R. Dahlen, J. R. Pursley, N. M. Bello, P. M. Fricke, M. C. Wiltbank, D. J. Brusveen, M. Burkhart, R. S. Youngquist, and H. A. Garverick. 2008. Detection of anovulation by heatmount detectors and transrectal ultrasonography before treatment with progesterone in a timed insemination protocol. J. Dairy Sci. 91:2901-2915.

Tenhagen, B.-A., M. Drillich, R. Surholt, and W. Heuwieser. 2004. Comparison of timed AI after synchronized ovulation to AI at estrus: Reproductive and economic considerations. J. Dairy Sci. 87:85-94.

Thomas, C. V., M. A. DeLorenzo, D. R. Bray, R. N. Weldon, R. A. Bucklin, and J. G. Martin III. 1997. A stochastic economic analysis of large herringbone and parallel milking parlors. J. Dairy Sci. 80:2418-2428.

Zacharias, T. P., and A. H. Grube. 1984. An economic evaluation of weed control methods used in combination with crop rotation: A stochastic dominance approach. N. Cent. J. Agric. Econ. 6:113120. 